\title{
Efeito da Escala de Produção nos Resultados Econômicos da Produção de Leite B no Estado de São Paulo 1
}

\author{
Eli Antônio Schiffler ${ }^{2}$, Antônio Bento Mâncio ${ }^{3}$, Sebastião Teixeira Gomes ${ }^{4}$, Augusto César de Queiroz ${ }^{3}$
}

\begin{abstract}
RESUMO - Este estudo avaliou os índices zootécnicos e produtivos, os custos de produção e a rentabilidade de quatro sistemas de produção de leite tipo B do Estado de São Paulo, que usam o sistema de pastejo intensivo como alimentação volumosa do rebanho no período do verão. Os quatro sistemas selecionados foram: sistema EMBRAPA/CPPSE, dois sistemas de produção particulares da região de São Carlos e um sistema localizado em São João da Boa Vista. O critério para escolha dos sistemas particulares foi ter perfis tecnológico e produtivo semelhantes aos do sistema EMBRAPA. Os dados coletados incluíram os índices zootécnicos e os recursos disponíveis de cada sistema. Obtidos os índices zootécnicos, os rebanhos foram estabilizados para permitir análises descritivas das características estudadas nos sistemas. Os resultados obtidos permitiram concluir que alta produtividade nem sempre significa maior lucro e que a escala de produção influenciou o custo total do litro de leite e, portanto, o lucro dos sistemas.
\end{abstract}

Palavras-chave: análises, comparação entre sistemas, eficiência técnica e econômica, índices zootécnicos, produtivos e econômicos, sistemas de produção

\section{Effect of Scale Production on Economic Results of Type B Milk Production in São Paulo State}

\begin{abstract}
This study evaluated the zootechnic and productive indexes, the production costs and the profit of four type B milk production systems in the State of São Paulo, Brazil, that use intensive pasture grazing as the forage source for the cattle during the summer. The four selected systems were: EMBRAPA/CPPSE system and two private production systems of São Carlos region, and one system located in São João da Boa Vista city. The criterion for the private systems selection was to have similar technology and productive profiles as the EMBRAPA system. The collection data included zootechnical index and available recourses for every system. Once the zootechnical index was obtained, the herd was stabilized to allow descriptive analyses of studied characteristics in the systems. The obtained results allowed to conclude that high productivity does not mean greater profit and that the production scale influenced the total cost per litre of milk and, therefore, the profit of the systems.
\end{abstract}

Key Words: analyses, comparisons among systems, technique and economic efficiencies, zootechnic, productive and economic indexes, production systems

\section{Introdução}

A abertura do comércio internacional, após a extinção de longo período de tabelamento do preço do leite, colocou o produtor brasileiro frente a fortes concorrentes de mercado, como os de Nova Zelândia e Argentina, detentores de baixos custos de produção e alta produtividade.

No Estado de São Paulo, os produtores de leite concorrem ainda com os de outros Estados, principalmente da região centro-oeste brasileira, além de concorrer internamente com atividades agrícolas como a canade-açúcar e a laranja (GOMES,1995). O baixo desempenho da pecuária leiteira estadual, com o correspondente desestímulo na atividade, tem diminuído a produção paulista a partir de 1993 e elevado o volume de leite importado (BORTOLETO et al., 1996).

Na região de São Carlos, SP, BARBOSA et al. (1989), em trabalho visando melhor conhecer a realidade da produção leiteira, verificaram que os produtores de leite $\mathrm{B}$ apresentaram médias de produção e produtividade bem superiores aos do leite C. Mesmo assim, após 1993, houve queda de produção também de leite B. Diante da atual situação, é necessário saber da viabilidade de se produzir leite no Estado de São Paulo. Para tanto, é de grande importância conhecer os índices técnicos e a lucratividade que atualmente compõem os sistemas de produção de leite tipo B.

A produção leiteira no mundo tem se elevado via inovação tecnológica, principalmente nas áreas de nutrição, melhoramento genético e saúde animal.

\footnotetext{
1 Parte da Tese de Mestrado do primeiro autor.

2 Eng. Agr., M.Sc., EMBRAPA/CPPSE, Caixa Postal 339 - 13560-970 - São Carlos, SP.

${ }^{3}$ Professor da Universidade Federal de Viçosa, Departamento de Zootecnia.

${ }^{4}$ Professor da Universidade Federal de Viçosa, Departamento de Economia Rural.
} 
Segundo FARIA e CORSI (1988), os produtores eficientes procuram intensificar a produção explorando melhor os recursos disponíveis, tornando a atividade mais competitiva, em relação a outras atividades econômicas da região. A intensificação da produção, seja por intermédio da produção de leite a pasto ou "free-stall", visa elevar a eficiência e a economicidade da exploração, por meio do melhor uso dos recursos técnicos, administrativos e financeiros. Dentre os diversos fatores responsáveis por exploração leiteira eficiente, destaca-se a alimentação adequada, que possibilita exprimir o potencial genético produtivo dos animais; esses, quanto mais produtivos, mais exigentes serão em nutrição e, portanto, em alimentação.

CAMARGO (1993) considera a elevação dos custos de produção como o fator responsável pelo abandono da atividade leiteira. Para que haja lucro, deve-se melhorar a eficiência produtiva, por meio da elevação da produção e produtividade. Segundo HOLMES et al. (1989), os custos são sensivelmente diminuídos quando se consegue manter rebanhos produtivos a pasto, utilizando recursos naturais forrageiros.

GOMES (1997), analisando empresas produtoras de leite consideradas eficientes no Estado de São Paulo, concluiu que leite é bom negócio quando elevados volumes de produção decorrem de elevados índices de produtividade. GALETTO (1996) concluiu haver diferença nos resultados econômicos em decorrência do efeito escala de produção e da habilidade administrativa na racionalização dos fatores produtivos.

Diante do exposto, este trabalho foi realizado com o objetivo de avaliar os índices produtivos, a produção e a viabilidade econômica de sistemas de produção de leite que utilizam o pastejo intensivo no verão, como única fonte de volumoso para o gado.

\section{Material e Métodos}

Considerou-se na seleção dos sistemas de produção aqueles que fossem de leite tipo B e que mais se assemelhassem aos perfis técnicos e produtivos do sistema da EMBRAPA - Pecuária Sudeste situado na região de São Carlos, SP. Com o auxílio de técnicos da região e dos dados de recepção leiteira da COLASC Cooperativa de Laticíonios de São Carlos, foram selecionados primeiramente sistemas quanto ao volume de produção diária e que tinham a alimentação volumosa do rebanho, no verão, por meio do pastejo rotacionado intensivo. Outras características gerais de semelhança foram consideradas na amostragem: topografia plana/ondulada, fertilidade original do solo médio, facilidade de acesso, telefonia, duas ordenhas mecânicas diárias, inseminação artificial e maquinário suficiente para a execução das tarefas rotineiras.

Os rebanhos selecionados eram semelhantes tanto nos aspectos produtivo e reprodutivo (Tabela 3), como no padrão genético (acima do grau de sangue 7/8 holandes-zebu), em que todos faziam seleção voltada para a apuração da raça holandesa. A suplementação concentrada foi feita o ano inteiro, de acordo com a produção e a suplementação volumosa com silagem de milho no período seco. Os lotes de recria foram divididos em função do peso dos animais. O controle sanitário e os manejos das pastagens e dos animais eram superiores à maioria dos produtores da bacia leiteira da região de São Carlos. Devido à impossibilidade de se selecionarem três sistemas particulares nesta região, selecionouse na região vizinha de São João da Boa Vista o terceiro sistema particular, que, incluindo o sistema EMBRAPA - Pecuária Sudeste, totalizou quatro sistemas. Os dados coletados para análise compreenderam o período de maio de 1995 a abril de 1996.

Todos os levantamentos dos quatro sistemas seguiram o mesmo roteiro utilizado por GOMES (1997), iniciando-se com os índices zootécnicos (intervalo de partos, doses de sêmen por concepção, período de lactação, idade à primeira cobrição, idade ao primeiro parto, porcentagem de vacas lactantes pelo total de vacas, porcentagem de vacas lactantes pelo total do rebanho, natalidade, mortalidade, raça e grau do sangue) e recursos disponíveis (uso das terras, benfeitorias, máquinas, animais, mão-de-obra, tecnologia adotada, dados de produção e demais informações complementares). Os dados zootécnicos e contábeis foram obtidos por meio dos registros existentes nas propriedades.

Após o levantamento dos índices zootécnicos, os rebanhos dos sistemas foram estabilizados (em termos de número de animais), para possibilitar comparações. Para a conferência e ajuste, quando necessário, dos índices zootécnicos após a estabilização, adotaram-se as seguintes relações:

\% de vacas em lactação = taxa de natalidade x período de lactação 365 dias

ou \% de vacas em lactação = período de lactação x 100 intervalo de partos

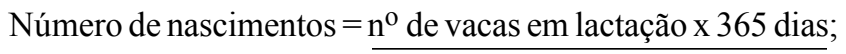
período de lactação

Número total de vacas $=\mathrm{n}^{\mathrm{o}}$ de nascimentos $\mathrm{x} 100$; e taxa de natalidade

Número de vacas secas $=\mathrm{n}^{0}$ total vacas $-\mathrm{n}^{\mathrm{O}}$ vacas lactantes. 
O uso das relações acima foram importantes para se aferir a exatidão das anotações zootécnicas realizadas pelos sistemas. Os índices zootécnicos dos quatro sistemas encontram-se na Tabela 2.

Os índices zootécnicos foram utilizados para cal- cular os índices produtivos e econômicos, que são os indicadores estudados neste trabalho.

No descarte anual de vacas, considerou-se venda de $20 \%$, sendo que $50 \%$ foram para abate e $50 \%$ para produção. A faixa etária de vendas foi de acordo com o critério de cada produtor.

Tabela 1 - Recursos disponíveis de quatro empresas produtoras de leite do Estado de São Paulo Table 1 - Available resourses of four dairy farms in the State of São Paulo

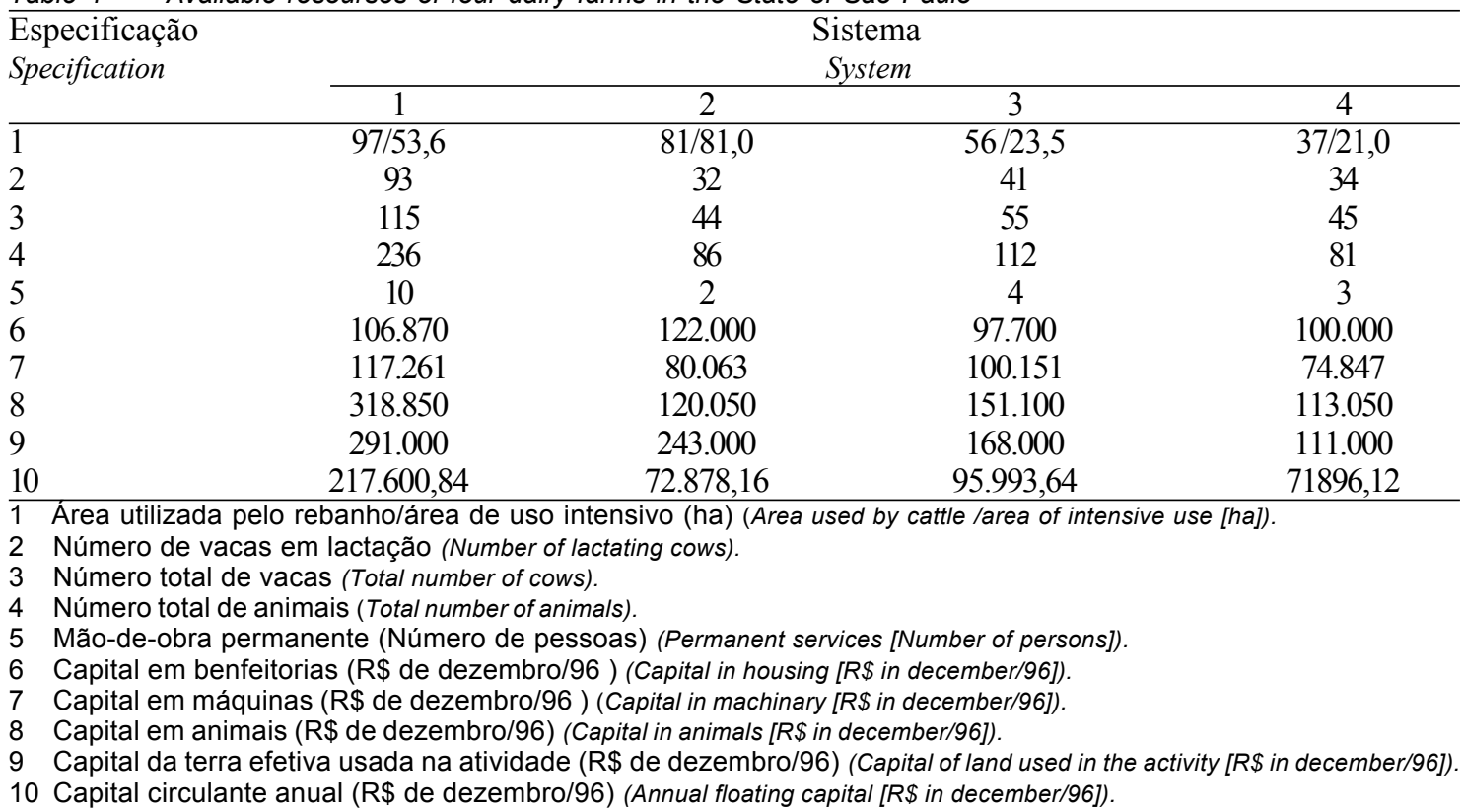

Tabela 2 - Índices zootécnicos nos quatro sistemas produtores de leite tipo B no Estado de São Paulo (dados referentes ao período de 1995-1996)

Table 2 - Zootechnical indexes of the four systems to produce type B milk in São Paulo state (figures reffer to 9596 period)

\begin{tabular}{|c|c|c|c|c|c|}
\hline \multirow{2}{*}{\multicolumn{2}{|c|}{$\begin{array}{l}\text { Especificação } \\
\text { Specification }\end{array}$}} & \multicolumn{4}{|c|}{$\begin{array}{l}\text { Sistema } \\
\text { System }\end{array}$} \\
\hline & & 1 & 2 & 3 & 4 \\
\hline 1 & & 382 & 411 & 385 & 420 \\
\hline 2 & & 99 & 128 & 102 & 137 \\
\hline 3 & & 2,68 & 1,60 & 1,70 & 1,41 \\
\hline 4 & & 309 & 299 & 287 & 318 \\
\hline 5 & & 350 & 330 & 330 & 400 \\
\hline 6 & & 549 & 549 & 488 & 610 \\
\hline 7 & & 80,90 & 72,70 & 74,50 & 75,50 \\
\hline 8 & & 39,41 & 37,21 & 36,61 & 41,98 \\
\hline 9 & & 95,6 & 88,6 & 94,5 & 86,7 \\
\hline 10 & & 1,8 & 5,3 & 2,0 & 5,3 \\
\hline 11 & & 27,4 & 28,0 & 25,5 & 30,0 \\
\hline \multicolumn{6}{|c|}{1 Intervalo de partos (dias) (Calving interval [days]). } \\
\hline 2 & \multicolumn{5}{|c|}{ Período de serviço (dias) (Days open [days]). } \\
\hline 3 & \multirow{2}{*}{\multicolumn{5}{|c|}{ Inseminação artificial (doses/concepção) (Artificial insemination [doses/conception]). }} \\
\hline 4 & & & \multicolumn{3}{|c|}{ Período de lactação (dias) (Lactation lenght [days]). } \\
\hline 5 & \multicolumn{5}{|c|}{ Primeira inseminação (kg/PV) (First insemination [kg/LW)]. } \\
\hline 6 & \multicolumn{5}{|c|}{ Primeira inseminação (dias) (First insemination [(days]). } \\
\hline 7 & \multicolumn{5}{|c|}{ Vaca lactante/total de vacas (\%) (Milking cow/total cows [\%]). } \\
\hline 8 & \multicolumn{5}{|c|}{ Vaca lactante/total rebanho (\%) (Milking cow/total herd [\%]). } \\
\hline 9 & \multicolumn{5}{|c|}{ Natalidade/total de vacas $(\%)$ (Calving rate $[\%]$ ). } \\
\hline 10 & \\
\hline 11 & \multicolumn{5}{|c|}{$\begin{array}{l}\text { Mortalidade até um ano (\%) (Mortality up to one year [(\%]). } \\
\text { Idade ao primeiro parto (meses) (Age at first calving [months]). }\end{array}$} \\
\hline
\end{tabular}


Foram utilizadas as seguintes relações, para conhecimento dos índices de produção e produtividade (Tabela 3):

Produção anual de leite/área efetiva (ha) usada pelo rebanho;

Produção de leite por dia/total de vaca;

Produção de leite por dia/vaca em lactação;

Vacas em lactação/área efetiva (ha) usada pelo rebanho;

Produção da vaca em lactação/dia de intervalo de partos; e

Produção anual de leite/mão-de-obra permanente (serv.).

Apesar das semelhanças existentes, as quantidades e os insumos usados nos sistemas variaram conforme a tecnologia adotada, sendo, portanto, diferentes entre si. Os preços usados para os cálculos de custos e receitas foram os praticados no comércio de São Carlos em dezembro/1996 e os utilizados nas planilhas da FNP-Consultoria e Comércio (1996).

Os valores da terra, do leite e dos animais foram os mesmos para todos os sistemas, em decorrência das características semelhantes. Os preços pagos à mãode-obra foram os praticados pelos produtores particulares. Para o caso da EMBRAPA/CPPSE, adotou-se o preço médio regional. Os critérios adotados para os cálculos de taxas, depreciação e remuneração do fluxo de serviços foram iguais para os quatro sistemas.

Como a pecuária de leite tem produção conjunta de leite e animais, isolou-se o custo de leite ponderando-se os custos totais pelo mesmo percentual da composição de renda, ou seja, do custo total da atividade, diminuiu-se a venda de animais. Esse resultado, dividido pelo custo total da atividade, é o custo total só do leite. Dessa forma, as tabelas de indicadores econômicos totais dos sistemas puderam ser desdobrados em indicadores unitários por litro de leite, além de outras unidades (ha, vaca/lactação, vaca/total), referentes à atividade leiteira (leite + animais).

Com base nas tabelas de indicadores econômicos totais dos sistemas, os custos foram divididos em operacional efetivo - COE (ou capital circulante), operacional total - COT (onde se inclui as depreciações) e total - CT (inclui as remunerações dos capitais empatados), propiciando a interpretação e análise do período a ser analisado (curto, médio e longo prazos - Tabela 4).

As margens bruta (receita bruta menos COE) e líquida (receita bruta menos COT) e o lucro (receita bruta menos $\mathrm{CT}$ ) representam os resultados financeiros respectivos dos diversos custos diminuídos pela renda bruta. O mesmo raciocínio é válido para as remunerações do capital circulante, sem terra e com terra, que refletem resultados, respectivamente, a curto, médio e longo prazos (Tabelas 5, 6 e 7). Para o cálculo das respectivas remunerações, divide-se a margem líquida pelos capitais circulante, investido sem a terra e investido com a terra. Os resultados encontrados nas divisões são multiplicados por 100 e podem ser comparados com as taxas de aplicações bancárias.

Os índices produtivos e econômicos foram comparados por meio de análises descritivas e agrupados em tabelas, possibilitando a comparação e discussão dos sistemas.

\section{Resultados e Discussão}

Nos demais índices produtivos, os resultados encontrados nos quatro sistemas, de modo geral, foram

Tabela 3 - Produção e produtividade de quatro empresas produtoras de leite do Estado de São Paulo Table 3 - Production and productivity of four dairy farms in the State of São Paulo

\begin{tabular}{lcccc}
\hline $\begin{array}{l}\text { Especificação } \\
\text { Specification }\end{array}$ & \multicolumn{4}{c}{$\begin{array}{c}\text { Sistema } \\
\text { System }\end{array}$} \\
\cline { 2 - 5 } & 1 & 2 & 3 & 4 \\
\hline 1 & 1968 & 654 & 910 & 610 \\
2 & 7404 & 2946 & 5931 & 6018 \\
3 & 17,11 & 14,86 & 16,55 & 13,56 \\
4 & 21,16 & 20,44 & 22,20 & 17,94 \\
5 & 6538,44 & 6111,56 & 6371,40 & 5704,92 \\
6 & 196,76 & 356,91 & 227,50 & 03,33 \\
7 & 0,96 & 0,4 & 0,73 & 0,92 \\
\hline 1 Produção média de leite diária (Litros/dia) (Average daily milk production [Liters/day]). & \\
2 Produção por área (Litros por ano/ha) (Milk production per area [Liters/ha]). \\
3 Produção por total de vacas (Litros por dia/vaca) (Milk production per total of cows [Liters/cow•day]). \\
4 Produção por vaca em lactação (Litros por dia/vaca) (Milk production per milking cow [Liters per day/cow]). \\
5 Produção por lactação (Litros/vaca em lactação) (Milk production per lactation [Liters/milking cow]). \\
6 Produção por mão-de-obra permanente (Litros/dia-homem) (Milk production per service [Liters/service]). \\
7 Número de vacas em lactação por ha (Média no ano) (Number of milking cows per ha).
\end{tabular}


semelhantes (Tabela 3 ) e bem superiores às médias de produção diária dos produtores paulistas de leite tipo B (388 litros) e de produção por vaca em lactação (914 litros) (ZOCCAL, 1996).

Apesar da seleção de sistemas com perfis técnicos e produtivos semelhantes ao da EMBRAPA no período analisado, as produções foram diferentes. Os produtores particulares produziram, em média, 37\% do volume de leite da EMBRAPA. Nos demais índices produtivos, os resultados encontrados nos quatro sistemas, de modo geral, foram semelhantes e bem superiores às médias da maioria dos produtores paulistas (Tabela 3 ). Estes índices demonstram a importância dos sistemas intensificados na obtenção de elevadas produtividades (FARIA e CORSI, 1988; HOLMES, 1987), já que todos os sistemas adotam esses conceitos.

\section{Índices produtivos}

Na Tabela 3 são apresentados os principais resultados de produção e produtividade dos sistemas. A diferença no volume de produção de leite do sistema 1 em relação aos demais sistemas pode ter influenciado os resultados econômicos, conforme ressaltam GALETTO (1996) e GOMES (1997). Este sistema foi $323 \%$ maior que o de menor volume (sistema 4 ). O sistema 3 foi superior aos sistemas 2 e 4 em 39 e $49 \%$ respectivamente, embora tenha produzido $216 \%$ menos que o sistema 1.

Os sistemas 1, 2 e 3 apresentaram produções diárias por vaca em lactação bem semelhantes, com variação máxima de $9 \%$. O sistema 4 obteve o pior resultado (17,94 litros), ou seja, $81 \%$ da melhor produtividade (sistema $3=22,20$ litros). Como as relações vacas lactantes/total de vacas foram semelhantes entre os sistemas, o sistema 4 apresentou também a menor produção diária por total de vacas (13,56 litros).

Os sistemas 1, 2 e 3 apresentaram, ainda, produções por período de lactação bem semelhantes, com variações abaixo de $10 \%$, com média de 6.340 litros por lactação. O sistema 4 apresentou o pior desempenho (5705 litros). Dentre diversos os fatores que podem ter influenciado os resultados, FARIA e SILVA (1996) destacam o período ideal de lactação (305 dias), que propicia descanso nas funções das glândulas mamárias e no restabelecimento do estado nutricional da vaca. No sistema 4 esta situação não ocorreu, pois, apesar de o período de lactação ter sido de 318 dias, o intervalo de partos foi de 420 dias, o que possibilitou 102 dias de descanso, bem acima do recomendado (60 dias).

O maior número de vacas por área efetivamente usada $(0,96 \mathrm{cab} / \mathrm{ha})$ contribuiu para o sistema 1 apre-

Tabela 4 - Custos (R\$) médios de produção por litro de quatro empresas produtoras de leite do Estado de São Paulo

Table 4 - $\quad$ Average costs $(R \$)$ of milk production per liter of four dairy farms in the State of São Paulo

\begin{tabular}{lcccc}
\hline $\begin{array}{l}\text { Especificação } \\
\text { Specification }\end{array}$ & \multicolumn{4}{c}{$\begin{array}{c}\text { Sistema } \\
\text { System }\end{array}$} \\
\cline { 2 - 5 } & 1 & 2 & 3 & 4 \\
\hline 1 & 0,2477 & 0,2655 & 0,2375 & 0,2733 \\
2 & 0,2604 & 0,3193 & 0,2733 & 0,3126 \\
3 & 0,2791 & 0,3399 & 0,2922 & 0,3339 \\
\hline
\end{tabular}

1 Custo operacional efetivo (Effective operating cost).

2 Custo operacional total (Total operating cost).

3 Custo total (Total cost).

Tabela 5 - Renda $(R \$)$ e custos de produção de quatro empresas produtoras de leite do Estado de São Paulo

Table 5 - Income $(R \$)$ and production costs of four dairy farms in the State of São Paulo

\begin{tabular}{lcccc}
\hline \multirow{2}{*}{\begin{tabular}{l} 
Especification \\
\cline { 2 - 4 }
\end{tabular}} & \multicolumn{3}{c}{$\begin{array}{c}\text { Sistema } \\
\text { System }\end{array}$} \\
\cline { 2 - 4 } 1 & $303.240,84$ & 2 & 3 & 4 \\
2 & $217.600,84$ & $98.092,20$ & $140.614,00$ & $93.634,00$ \\
3 & $228.721,51$ & $72.878,16$ & $95.993,64$ & $71.896,12$ \\
4 & $245.126,80$ & $87.633,47$ & $110.452,08$ & $82.231,80$ \\
5 & $85.640,00$ & $93.299,72$ & $118.095,93$ & $87.813,23$ \\
6 & $74.519,33$ & $25.214,04$ & $44.620,36$ & $21.737,88$ \\
7 & $58.114,04$ & $10.458,73$ & $30.161,92$ & $11.402,20$ \\
7 Renda bruta da ativ. leiteira (Leite + animais) (Gross income of the dairy activity [milk + animals]). & 5820,77 \\
2 Custo operacional efetivo da atividade leiteira (Effective operating cost of the dairy activity). & \\
3 Custo operacional total da atividade leiteira (Total operating cost of the dairy activity). & \\
4 Custo total da atividade leiteira (Total cost of the dairy activity). & \\
5 Margem bruta da atividade leiteira (Gross margin of the dairy activity). & \\
6 Margem líquida da atividade leiteira (Net margin of the dairy activity). & \\
7 Lucro da atividade leiteira (Profit of the dairy activity). &
\end{tabular}


Tabela 6 - Lucro (R\$) de quatro empresas produtoras de leite do Estado de São Paulo

Table 6 - Profit (R\$) of four dairy farms in the State of São Paulo Especificação Sistema

Specification $\quad$ System

\begin{tabular}{lcrrr}
\cline { 2 - 5 } & 1 & \multicolumn{4}{c}{3} & \multicolumn{1}{c}{4} \\
\hline 1 & 0,0809 & 0,0201 & 0,0678 & 0,0261 \\
2 & 599,11 & 59,17 & 402,11 & 157,32 \\
3 & 505,34 & 108,92 & 409,42 & 129,35 \\
4 & 624,88 & 149,77 & 549,22 & 171,20 \\
\hline
\end{tabular}

1 Lucro/litro de leite (Profit per liter of milk).

2 Lucro anual/ha usado pelo rebanho (Anual profit per hectare used by the herd).

3 Lucro anual por total de vacas (Anual profit per total number of cows).

4 Lucro anual por vacas em lactação (Anual profit per milking cows).

Tabela 7 - Taxas anuais de remuneração (\%) do capital de quatro empresas produtoras de leite do Estado de São Paulo

Table 7 - $\quad$ Anual rates of gain (\%) of capital of four dairy farms in State of São Paulo

\begin{tabular}{lrrrr}
\hline Especificação & \multicolumn{4}{c}{ Sistema } \\
Specification & \multicolumn{4}{c}{ System } \\
\cline { 2 - 5 } & \multicolumn{1}{c}{2} & \multicolumn{1}{c}{3} \\
\hline 1 & 34,25 & 14,35 & 31,42 & 15,86 \\
2 & 9,80 & 2,65 & 6,78 & 3,17 \\
3 & 7,09 & 1,64 & 4,92 & 2,42 \\
\hline
\end{tabular}

1 Remuneração do capital circulante (\% ao ano) (Remuneration of the floating capital [\% in the year]).

2 Remuneração do capital (Sem terra - \% ao ano) (Remuneration of the capital [No land - \% in the year]).

3 Remuneração do capital (Com terra - \% ao ano) (Remuneration of the capital [With land - \% in the year]).

sentar maior lucratividade, haja visto a semelhança que os outros índices de produtividade obtiveram entre sistemas; ainda assim o sistema 1 está operando abaixo do seu potencial. GOMES (1997) aponta entre 2 a 3 vacas em lactação/ha/ano como o ideal para as condições paulistas. Caso se considerasse apenas a área de uso intensificado (área para volumosos e pastejo intensivo), os sistemas 1, 3 e 4 praticamente duplicariam estes resultados. A taxa de lotação é importante, pois reflete a capacidade de suporte e a eficiência do pastejo intensivo de cada sistema.

Nos levantamentos dos sistemas 2 e 3 foi verificado o uso de silagem no período de novembro/95 a janeiro/96, para as vacas em lactação que deveriam estar pastejando. Esta prática denota manejo irregular das pastagens, comprometendo os resultados econômicos. Estes dois sistemas apresentaram as piores taxas de lotação.

A produção de leite por mão-de-obra permanente apresentou o sistema 2 como o de melhor desempenho, sendo $181 \%$ mais eficiente que o sistema 1 , que foi o pior, com 197 litros/serviço. É interessante frisar que o sistema 2 foi o único em que o produtor residia e administrava diretamente a atividade. O sistema 1 (EMBRAPA) foi o pior, porque, provavelmente, os horários de trabalho são mais respeitados em uma empresa estatal e os funcionários, além da produção, têm afazeres na pesquisa e no atendimento a visitantes e estagiários. De modo geral, os índices estão abaixo do ideal. Como os sistemas já apresentam razoável uso de mecanização e as diversas tarefas rotineiras dificultam melhor racionalizar a mão-deobra, a melhoria desse índice depende mais do aumento no volume da produção leiteira dos sistemas.

\section{Índices econômicos}

$\mathrm{Na}$ Tabela 4 estão apresentados os custos médios por litro de leite dos quatro sistemas. Os sistemas 1 e 3 apresentam maior produção que os 2 e 4 no custo operacional efetivo.

Quando se compara o volume de produção de leite de cada sistema, com o capital imobilizado em terras, benfeitorias e máquinas (Tabela 1), os sistemas 1 e 3 apresentam maior produção em relação aos 2 e 4. Esta diferença é mais perceptível nos custos operacionais totais e custos totais, o que significa que os sistemas 2 e 4 têm custos de depreciação e remuneração de capital mais elevados ou menos "diluídos" pelo volume de produção, refletindo assim em maiores custos totais por litro de leite.

$\mathrm{Na}$ Tabela 5 pode-se observar que os custos, bem como a margem bruta, foram compatíveis com os volumes de produção apresentados pelos sistemas, ou seja, quanto maior a produção, maior a margem bruta.

A margem líquida e o lucro do sistema 2 foram os piores e assemelharam-se aos do sistema 4. Ambos tiveram os maiores custos de depreciação e de remuneração de capitais. Os sistemas 1 e 3, apesar de apresentarem custos, em geral, mais elevados (Tabela 5), tiveram margens bruta, líquida e lucro superiores, resultantes do efeito de escala de produção . O sistema 1 apresentou o maior lucro (1.213\% maior que o sistema 2 - Tabela 5), demonstrando que os resultados econômicos são reflexos dos resultados produtivos, que por sua vez traduzem os resultados zootécnicos.

É interessante observar que os índices produtivos entre sistemas apresentaram pequenas variações. As exceções são o número de vacas em lactação e a produtividade por área. Apesar do sistema 4 apresentar taxa de lotação semelhante ao sistema 1, apresentou também os piores índices zootécnicos e produtivos e o menor volume de produção, o que 
comprometeu sua eficiência econômica.

Na Tabela 7, os resultados demonstraram que os sistemas cobriram os custos do capital circulante, estando bem acima do valor da caderneta de poupança (6\% ao ano).Todavia, na remuneração do patrimônio capital sem terra, os sistemas 2 e 4 foram deficitários, quando comparados com a rentabilidade da poupança. Isso significa que esses sistemas terão dificuldades em repor o capital imobilizado em máquinas, benfeitorias e forrageiras não anuais, quando findarem a vida útil das mesmas.

Em remuneração do patrimônio capital com terra, além dos resultados desprezíveis dos sistemas 2 e 4, o sistema 3 situou-se pouco abaixo da remuneração da poupança, demonstrando que o lucro obtido não remunerou o patrimônio imobilizado com a terra.

Assim, caso não ocorra melhoria nas eficiências econômicas, os sistemas 2 e 4 a médio prazo e o sistema 3 a longo prazo serão mais lucrativos, se aplicarem os seus recursos disponíveis no mercado financeiro. Este resultado sinaliza a necessidade de se elevarem a eficiência produtiva e o volume de produção, como forma de se reduzirem os custos.

Algumas medidas podem ser tomadas nos quatro sistemas para torná-los mais competitivos por intermédio da elevação da produção leiteira:

O sistema 2 deve melhorar o manejo das pastagens e da alimentação, aumentar o plantel de matrizes e, portanto, o número de vacas lactantes e a taxa de lotação das pastagens, via aumento da capacidade de suporte, e melhor racionalizar o uso dos demais recursos disponíveis.

O sistema 4 deve melhorar os índices produtivos via melhor desempenho zootécnico, aumentar o plantel de matrizes e a área intensificada da atividade leiteira e melhor racionalizar o uso dos recursos disponíveis.

O sistema 3 necessita melhorar principalmente o manejo das pastagens. A maior eficiência, além de eliminar custos de suplementação volumosa no verão, irá elevar a taxa de lotação, em razão do aumento da capacidade de suporte. A seleção de matrizes mais especializadas, de maior "grau racial" Holandês, deverá se basear em aspectos produtivos, principalmente na maior persistência de lactação.

O sistema 1, da EMBRAPA/CPPSE, pode tornar-se ainda mais eficiente, via elevação do efeito escala, pela racionalização constante no uso dos recursos disponíveis, pela ampliação da área de pastejo intensivo, pelo aumento da capacidade de suporte e pela melhoria dos índices zootécnicos. Por pertencer à EMBRAPA, o que permite incorporação constante de novas tecnologias, o sistema 1 tem a necessidade e as condições básicas de elevar ainda mais suas eficiências e continuar servindo de polo irradiador de técnicas a serem usadas pelo produtor rural.

\section{Conclusões}

Alta produtividade não significa sempre maior benefício econômico.

A produção de escala de leite tem influência no custo total do litro de leite.

Análises de viabilidade produtiva de sistemas de produção necessariamente devem compor análises de custos e receitas.

Estudos semelhantes poderão ser importantes para estabelecimento de volumes de produções econômicas de sistemas em uma região e na detecção dos seus pontos de estrangulamento.

\section{Referências Bibliográficas}

BARBOSA, P.F., COSTA, J.L., CRUZ, G.M. et al. 1989. Acompanhamento e avaliação de sistemas reais de produção de leite na região de São Carlos. São Carlos, EMBRAPA/ UEPAE de São Carlos.

BORTOLETO, E.E., CROCETTA, I., RAMOS, J. et al. 1996. Repensando a agricultura paulista: Cadeia produtiva do leite no Estado de São Paulo. São Paulo: Secretaria da Agricultura e Abastecimento, 61p.

CAMARGO, A.C. Planejamento de fazendas leiteiras para intensificação do processo produtivo através do uso de pastos de capim elefante. In: SIMPOSIO SOBRE MANEJO DE PASTAGEM, 1, 1993, Piracicaba. Anais... Piracicaba: FEALQ, p. 277-293, 1993.

FARIA, V.P., CORSI, M. 1988. Índices de produtividade em gado leiteiro. In: PEIXOTO, A. M. et al. Produção de leite: conceitos básicos. Piracicaba: FEALQ, p.23-44.

FARIA, V.P., SILVA, S. C. Fatores biológicos determinantes na pecuária leiteira. In: SIMPÓSIO INTERNACIONAL - O FUTURO DOS SISTEMAS DE PRODUÇÃO DE LEITE NO BRASIL, 1996. Juiz de Fora, MG, p.77-89, 1996.

FNP. Consultoria e Comércio. 1996. Anuário estatístico da produção animal: ANUALPEC 96. São Paulo: Argos, 312p.

GALETTO, A. 1996. Um enfoque preliminar sobre el resultado econômico de la intensificacion de la producion lechera. Desarollo Rural, n.58.

GOMES, S.T. 1995. Análise histórica e perspectivas da oferta e da demanda do leite do Brasil. Viçosa: UFV, 12 p.

GOMES, S.T. 1997. A viabilidade do leite em São Paulo. Leite $B, 11(123): 10-21$.

HOLMES, C.W. 1989. Produção de leite a pasto. Campinas: Instituto Campineiro de Ensino Agrícola.

ZOCCAL, R.1994. Leite em números. Coronel Pacheco, MG: EMBRAPA - CNPGL, Belo Horizonte, MG: FAEMG, 131p.

Recebido em: 03/12/97 Aceito em: 02/10/98 